\title{
ARTICLE
}

\section{Development of Neutron-Gamma Discrimination Technique using Pattern-Recognition Method with Digital Signal Processing}

\author{
D. TAKAKU, T.OISHI, M.BABA* \\ Cyclotron and Radioisotope Center, Tohoku University \\ Aramaki, Aoba-ku, Sendai 980-8578, Japan
}

\begin{abstract}
We have developed a new neutron-gamma $(n-\gamma)$ pulse shape discrimination technique, a pattern-recognition method, for an NE213 scintillation detector by using digital signal processing. In this method, a signal to be analyzed is digitized and regarded as a vector, and the $n-\gamma$ discrimination is performed by taking a scalar product of the signal with a reference wave form vector to see the similarity of the pulse shape. We applied the method to an NE213 scintillation detector, and compared the results with those by the conventional curve-fitting and charge-integration methods. The pattern-recognition method proved to achieve best neutron- $\gamma$ separation with short data processing time comparable with the charge-integration method. This technique will be applied to various applications including pulse-shape analysis even in real time operation owing to quick data processing.
\end{abstract}

\section{KEYWORDS: n- $\gamma$ discrimination, NE213, digital-signal-processing, pattern recognition method, good separation, short computation time}

\section{Introduction}

The neutron-gamma $(\mathrm{n}-\gamma)$ pulse-shape discrimination (PSD) technique is of crucial importance in radiation measurement and dosimetry ${ }^{1)}$. For example it is indispensable in neutron measurements using liquid organic scintillation detectors like NE213 or gas counters to carry out reliable measurement by rejecting $\gamma$-ray backgrounds accompanied with the neutron fields. Besides, by applying the $\mathrm{n}-\gamma$ discrimination technique, it is possible to measure the spectra of neutrons and $\gamma$-rays concurrently in a single measurement.

The $n-\gamma$ discrimination is based on the fact that pulseshapes of radiation detectors are different according to the stopping power of particles ${ }^{1)}$. In the case of the NE213 scintillation detector, the decay time of the scintillation light is different between neutrons and $\gamma$-rays as shown in Fig. $1^{1)}$ because of different specific ionization of protons and electrons produced by neutrons and $\gamma$-rays, respectively.

Various $\mathrm{n}-\gamma$ discrimination techniques have been developed using hard-wired circuits, i.e., the rise-time method $^{2),}$ zero-crossing method ${ }^{3)}$ and the charge-integration method $^{4,5)}$ etc. They have been applied extensively under various conditions.

However, their performances are not enough for low energy neutrons $\mathrm{s}^{2-5)}$, and they are difficult to operate under a high count-rate with signal pileups.

Recently, a digital signal processing (DSP) technique can be

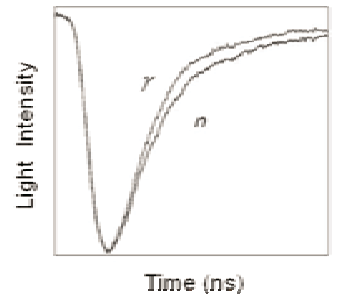

Fig. 1 Pulse-shape difference in an NE213 scintillator
*Corresponding Author, Tel:+81-22-795-7805, Fax. +81-22-795-7 809, E-mail:babam@cyric.tohoku.ac.jp, applied to various problems $\mathrm{s}^{6-11)}$ owing to the development of high speed and high resolution flash-type analogue-to-digital convertors (ADCs) and memories. In DSP, a signal wave form is converted into digital data and stored in memories, and the stored digital data are analyzed with computer. Then, DSP enables us to acquire almost all the information of signals, i.e., energy, time, pulse-shape, and complicated data analysis that is not accessible in the hard-wired systems ${ }^{1,6-11)}$ For example, Marrone et al. ${ }^{7,8}$ and Itoga et al. ${ }^{10,11)}$ succeeded $\mathrm{n}-\gamma$ PSD in scintillation detectors under pile-up conditions by fitting the digital wave form data with curves describing an isolated neutron or $\gamma$-ray signal. Generally, however, data processing in DSP is time taking because of a large volume of data. It is desired, therefore, to develop a fast data analysis method for effective utilization of DSP.

In the present study, we report a new $n-\gamma$ PSD technique which employs a pattern-recognition method ${ }^{12)}$. This method regards the digitized wave form data of interest as a vector and takes a scalar-product of this vector with a reference vector of the pulse-shape. Short processing time and simple procedure are the advantages of the method. Sakai et al., applied successfully the technique to the pulse-shape analysis of the analogue data from a CdZnTe detector ${ }^{12)}$.

In the present study, we applied the method to the $\mathrm{n}-\gamma$ discrimination in NE213 using a fast digital oscilloscope, and compare the results with those of the conventional curve-fitting and charge-integration methods. The patternrecognition method proved to be best in discrimination capability and data processing time.

\section{Methods}

The schematic view of the data acquisition system is shown in Fig.2. It was consisted of a neutron detector (NE213), a digital oscilloscope (DSO: LeCroy Wavepro 7000) and personal computer (PC). The NE213 neutron 
detector was 2 " in diameter and 2 " thick, coupled to a fast photomultiplier assembly ${ }^{6,7)}$, Hamamatsu H1949. The signal from the anode of the NE213 detector was fed directly into DSO which had a sampling rate of $10 \mathrm{G}$ samples/s, 8 bits resolution and 4 channel inputs.

The experimental data were taken at the Fast Neutron Laboratory, Tohoku University ${ }^{13)}$ for neutrons obtained with the $\mathrm{D}(\mathrm{d}, \mathrm{n})$ reactions using a $\mathrm{D}_{2}$ gas target ${ }^{13)}$. The nominal energy of neutrons was $5 \mathrm{MeV}$.

The pulse shape was digitized with $0.1 \mathrm{~ns}$ sampling time and data were taken for 2,000 points $(200 \mathrm{~ns})$ for each signal, and accumulated up to 50,000 counts. The low limit of the pulse height was around $0.7 \mathrm{MeV}$ of protons. To observe clearly the pulse-shape separation down to low pulse-height signals, data were taken in two gains, i.e., high-gain and low-gain. The gain ratio of the high-gain to low-gain system was about 1.5. The pulse height spectrum of neutrons show a rectangular-like shape as expected.

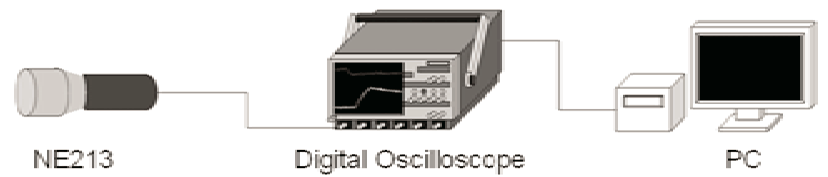

Fig.2 Schematic view of data acquisition system consisting of NE213 neutron detector, digital oscilloscope Wavepro 7000, and personal computer (PC).

\section{Analysis and Results}

The digital data obtained with DSO were analyzed for the pulse-shape using the pattern-recognition method as well as the curve-fitting and charge-integration methods. Prior to the pulse-shape analysis, each signal was pre-processed with the three-point moving-average method to reduce the contribution of high frequency noises as shown in Fig.3. The smoothing was made over 20 points. Then, the data were analyzed for pulse-shape with three methods adopting the MATLAB language ${ }^{14,15)}$.

For PSD, usually, a two-dimensional scatter-plot of the shape-parameter vs the total-charge or pulse-height is used because the shape-parameter depends on the total-charge or pulse-height of particles. In the present study, we used the

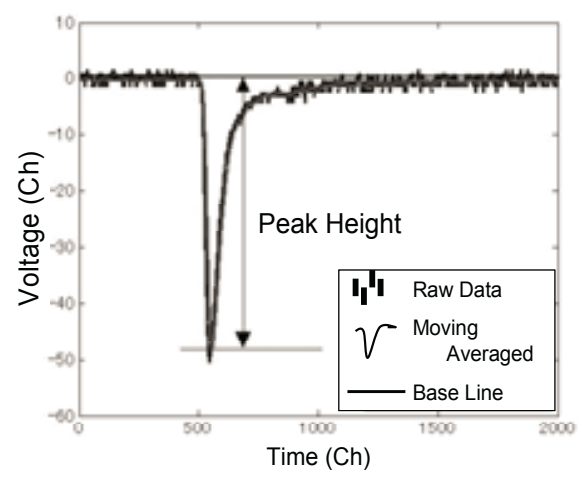

Fig.3 Pre-processing of signals with a moving average method. peak-height (Fig.3) in place of the total-charge or pulseheight in order to reduce the computation time or the number of data processing. The base line was determined from the average over 300 points in front of the signal. We confirmed that the result of this method did not differ appreciably from that using the total-charge.

In the followings, the method and the results are shown for the charge-integration, curve-fitting and the patternrecognition methods. The results are compared in terms of the PSD performance and the computation time.

\section{Charge-Integration Method ${ }^{4,5)}$}

The schematic view of the method is shown in Fig.4. In this method, the pulse-shape parameter is the charge in the tail region and noted as "area" below. The area is obtained by the integration of the signal with time over $\mathrm{T}$ as shown in Fig.4. A scatter-plot of the area vs the peak-height is used for PSD. This technique is used widely not only in DSP but also in hard-wired circuits ${ }^{4,5)}$.

Typical results of two dimensional plot for pulse-shape vs peak-height are shown in Fig.5 for each of high-gain and low-gain. The figure indicates good separation down to around 30 channel in the high-gain system corresponding to around $800 \mathrm{keV}$ of protons. However, as an inherent problem of this method, neutron signals and $\gamma$-ray signals tend to overlap in the lower pulse-height region and the separation between them becomes unclear.

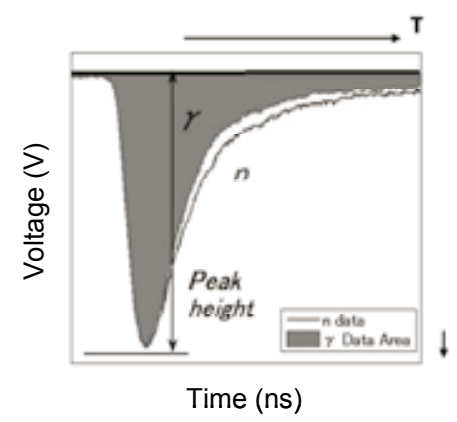

Fig.4 Scheme view of the charge-integration method
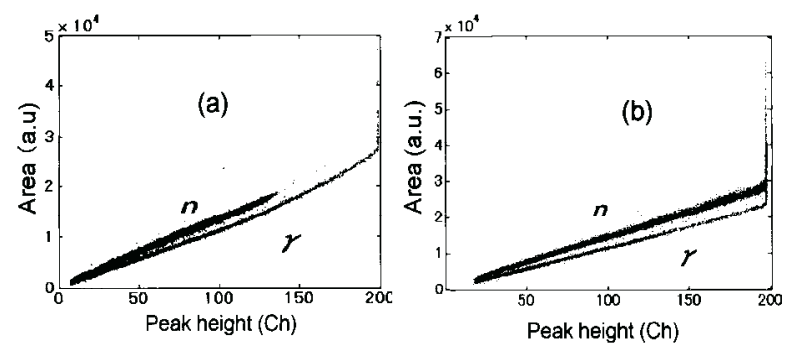

Fig.5 Scatter plots for area (arbitrary unit) vs peak-height in the charge-integration method; (a) low gain, (b) high gain

\section{Curve-Fitting Method ${ }^{8,9)}$}

In this method, the particle type is identified from the decay time of the pulse. In the present case, we obtained the decay time by fitting the tail part of the signal with the following single curve, 


$$
\mathrm{y}=\exp (-\lambda \mathrm{t})
$$

The decay constant $\lambda$ is the shape parameter, and a scatter plot of $\lambda$ vs peak-height gives the information for PSD. A typical fitting result is shown in Fig.6. In this figure, the experimental data deviate systematically from the fitted curve in the region above around 150 channel. It is known that better fit can be obtained by use of multiple decay components ${ }^{8,9)}$. Nevertheless, we found that the present one parameter fit provides sufficient results for $n-\gamma$ PSD as shown below.

The results of the curve-fitting method are shown in Fig.7. The $n-\gamma$ separation is much clearer than in the charge-integration method, and almost complete down to the lowest pulse-height signals ( $\sim 0.7 \mathrm{MeV}$ protons). Besides, in particular in high gain data, the pulse-shape parameter $\lambda$ is almost independent of the peak-height. Marrone et $\mathrm{al}^{8,9)}$ reported similar good results by the method. Such good separation by this method will partly due to reduction of noise contribution through the fitting procedure. As shown in the next section, however, this method needs long computation time even in the case of the present one parameter fit.

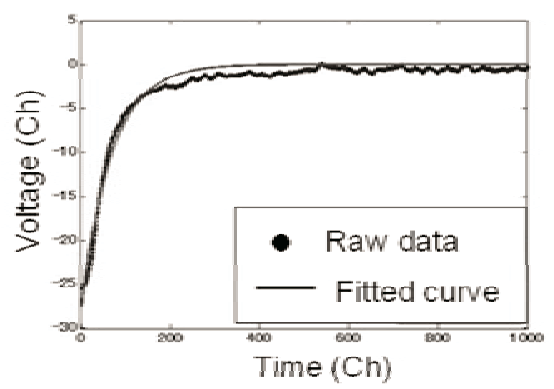

Fig.6 A typical result of fitting to the experimental data.
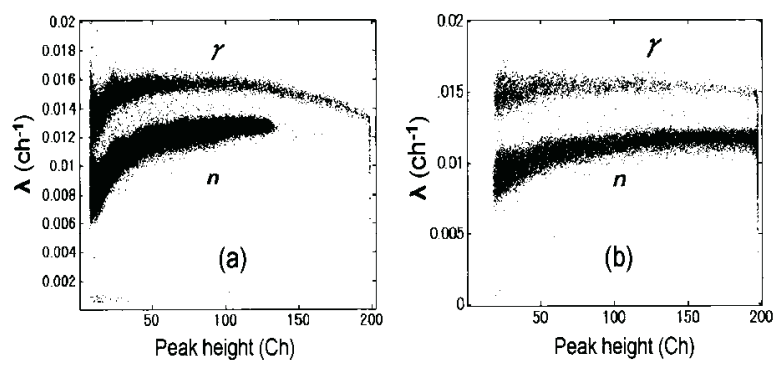

Fig.7 Scatter plots for $\lambda\left(\right.$ channel $^{-1}$ ) vs peak-height in the curve-fitting method; (a) low gain, (b) high gain.

\section{Pattern-Recognition Method ${ }^{12)}$}

In this method, a signal is regarded as an object vector $\boldsymbol{X}$ whose components are the digitized amplitude $x_{n}$ of the signal at sampling time $t_{n}(\mathrm{n}=1-2000)$. PSD is done by taking an scalar-product of this vector with the reference vector $\boldsymbol{Y}$ which describes a $\gamma$-ray or neutron signal.

$$
\begin{aligned}
& \mathbf{X}=\left[x_{1}, x_{2}, \ldots \ldots ., x_{2000}\right] \\
& \mathbf{Y}=\left[y_{1}, y_{2}, \ldots \ldots . ., y_{2000}\right]
\end{aligned}
$$

$$
\theta=\arccos \frac{\mathbf{X} \cdot \mathbf{Y}}{|\mathbf{X}||\mathbf{Y}|}
$$

where $\mathbf{X} \cdot \mathbf{Y}$ is the scalar product, $|\mathbf{X}|$ and $|\mathbf{Y}|$ are the norm of the vectors $\boldsymbol{X}$ and $\boldsymbol{Y}$, respectively, and $\theta$ is the angle between the vectors. The value of $\theta$ indicates the similarity of the object vector with the reference vector.

In the course of the present study, we found that the result of this method strongly depends on the selection of the reference signal, and better results are obtained by taking the $\gamma$-ray signal as the reference vector probably owing to lower dispersion of the $\gamma$-ray pulse-shape, and furthermore, good separation is obtained by taking the scalar product only for the tail region where the shape difference is enhanced.

The results of the pattern-recognition method are shown in Fig.8 for both low-gain and high-gain. In this case, the reference vector is a $\gamma$-ray signal, and the scalar-product is taken only for the tail region following the above mentioned observations. The separation is almost complete as shown by the valley between the neutron and $\gamma$-ray signals down to the lowest peak-height in high gain data.
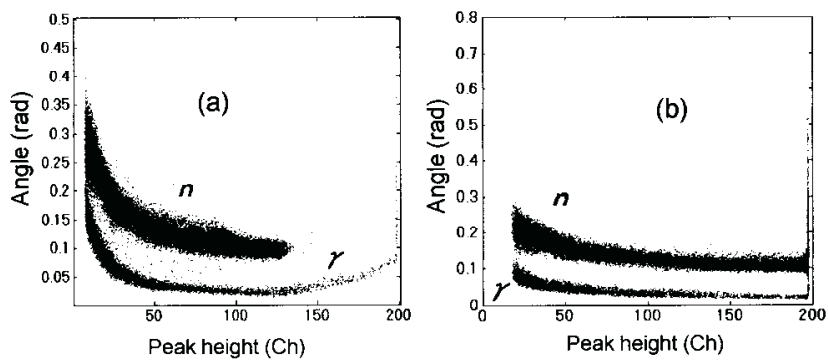

Fig.8 Scatter plots for angle (radian) vs peak-height in the pattern-recognition method; (a) low gain, (b) high gain.

\section{Quantitative Comparison of the Results}

For quantitative comparison of the quality of separation between $\gamma$-rays and neutrons by those three methods, we consider "Figure-of-Merits (FOM)" which is defined as ${ }^{16)}$

$$
\mathrm{FOM}=\left|\mathrm{Ch}_{\mathrm{n}}-\mathrm{Ch}_{\gamma}\right| /\left(\mathrm{FWHM}_{\mathrm{n}}+\mathrm{FWHM}_{\gamma}\right)
$$

where, $\mathrm{Ch}_{\mathrm{n}}$ and $\mathrm{Ch}_{\gamma}$ are, respectively the peak channel of seutron events and $\gamma$-rays events, and $\mathrm{FWHM}_{\mathrm{n}}$ and $\mathrm{FWHM}_{\gamma}$ are, respectively, the full width at half maximum of the neutron peak and the $\gamma$-ray peak in the $n-\gamma$ spectrum, which is the projection of the two dimensional scatter plot into the pulse-shape axis. Therefore, a larger FOM value means better separation between neutron and $\gamma$-ray events.

Figure 9 illustrates the FOM values as a function of the peak-height for the lower pulse-height region in low gain data. Table 1 compares the "Time" which is the computation time required to process 50,000 events by the method. 


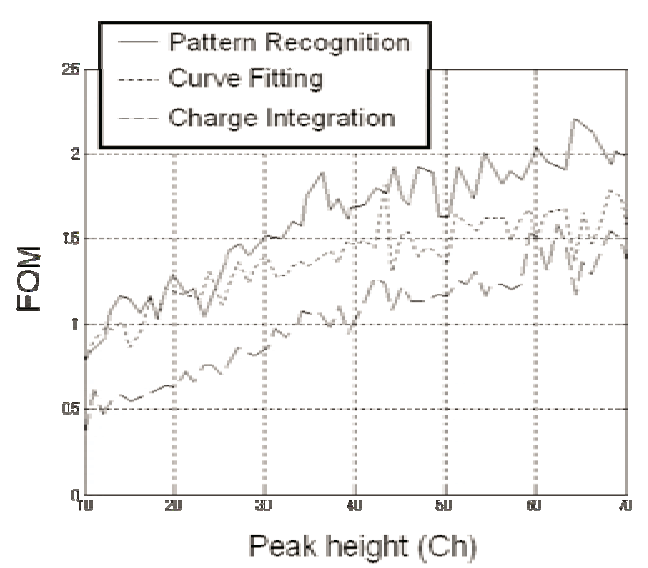

Fig.9 Dependence of FOM values on the peak-height for the pattern-recognition, curve-fitting and chargeintegration methods in lower pulse-height region of the low gain data.

Table 1: Computation time to complete the analysis of 50,000 events.

\begin{tabular}{|c|c|}
\hline Method & Time [s] \\
\hline Charge-Integration Method & 128 \\
\hline Curve-Fitting Method & 2086 \\
\hline Pattern-Recognition Method & 140 \\
\hline
\end{tabular}

Figure 9 and Table 1 indicate that all the PSD methods can separate neutron and $\gamma$-ray events satisfactorily over almost whole region as shown by the FOM values larger than one except for the case of the charge-integration method in lowest pulse-height region (below $\sim 1 \mathrm{MeV}$ ). Among the three methods, the pattern-recognition method gives the best FOM values trough the whole peak-height region. In particular, the FOM value of the method above $\sim 30$ channel is close to two which means complete separation with no overlapping.

Furthermore, this method is much faster than the curve-fitting method which gives comparable separation and is close to the charge-integration method. If multi-parameter fit is used in the curve-fitting method, time difference will be much larger. In the case of the pattern-recognition method, the average process time for each event is around 2.8 $\mathrm{ms} /$ event. That means that the method can be applied to the online analysis using the DSP technique, if count rate is in the range of hundreds per second.

In principle, this technique can be extended to various applications such as real time neutron and $\gamma$-dosimeter with PSD capability, and pulse-shape analysis not only for scintillation detectors but also for other detectors such as gas and/or solid state detector.

\section{Summary}

We have developed a $n-\gamma$ pulse-shape discrimination technique for an NE213 scintillation detector using the pattern-recognition method with the digital signal processing (DSP) technique. The method proved to achieve very good separation down to below $1 \mathrm{MeV}$ of protons with short computation time comparable to the conventional chargeintegration method. The pattern-recognition method, therefore, is very promising as a high quality and fast pulse-shape discrimination technique applicable to various pulse-shape analysis with on-line application.

\section{References:}

1) Glenn F.Knoll, "Radiation Detection and Measurement" $3^{\text {rd }}$ Ed. John Wiley \& Son, Inc. New York, 230,1999

2) V.T.Jordanov and G.F.Knoll, IEEE Trans. Nucl. Sci., 42 (4), 683 (1995)

3) D. Wolski, M. Moszynski, T. Ludziejewski, A. Johnson, W. Klamra, O. Skeppswtedt, Nucl. Instrum. Methods.,A360,584 (1995)

4) J. H. Heltsley, L. Brandon, A. Galonsky, L. Heilbronn, B. A. Remington, S. Langer, A. Vander Molen, J. Yurkon, J. kasagi, Nucl. Instrum. Methods.,A263, 441 (1988)

5) Y. Kaschuck and B. Esposito, Nucl. Instrum. Methods.,A551, 420 (2005)

6) T. Oishi, T. Yamauchi, M. Hagiwara, M. Baba, H. Yuki, T. Ohutsuki, J. Hori, Proc. Int. Symp. on Fast Neutron Detectors and its Application, April 2006, Proceedings of Science 2006;

7) Takuji Oishi and Mamoru Baba, Supplement-5 of J. Nucl. Sci. Technol., 375 (2008)

8) S. Marrone, D. Cano-Ott, N. Colonna, C. Domingo, F. Gramegna, E. M. Gonzalez, F. Gunsing, M. Heil, F. Kappeler, P. F. Mastinu, P. M. Milazzo, T. Papaevangelou, P. Pavlopoulos, R. Plag, R. Reifarth, G. Tagliente, J. L. Tain and K. Wisshak, , Nucl. Instr. and Meth., A490, 299 (2002)

9) S. Marrone, Nucl. Instrum. and Methods., B213, 246 (2004)

10) T. Itoga, M. Ishikawa, M. Baba, T. Okuji, T. Oishi, M. Nakhostine, T. Nishitani, Proc. Int. Symp. on $10^{\text {th }}$ Neutron Dosimetry, Radiation Protection and Dosimetry, 126 (No.1-4), 380 (2007)

11) K. Shinohara, M. Takechi, M. Ishikawa, Y. Kusama, A. Morioka, N. Oyama, K. Tobita, T. Ozeki, the JT-60 Team, N. N. Gorelenkov, C. Z. Cheng, G. J. Kramer and R. Nazikian, Nucl. Fusion, 42, 942, (2002)

12) H. Sakai, A. Uritani, Y. Takenaka, C. Mori, T. Iguchi, Nucl. Instrum. and Methods.,A421, 316 (1999)

13) M. Baba, M. Takada, T. Iwasaki, S. Matsuyama, T. Nakamura, H. Ohguchi, T. Nakao, T. Sanami, N. Hirakawa, Nucl. Instrum. Methods, A376, 115 (1996),

14) Y. Uesaka, "Introduction to MATLAB programming", Makino-shoten, Tokyo, 1, 2000, in Japanese

15) http://www.mathworks.com/

16) R. A. WINYARD, J. E. LUTKIN and G. W. McBETH,,Nucl. Instrum. and Methods.,95,141 (1971) 